\title{
Ester Derivatives of Kojic Acid and Polyphenols Containing Adamantane Moiety with Tyrosinase Inhibitory and Anti-inflammatory Properties
}

\author{
Ho Sik Rho, ${ }^{*}$ Myeongin Goh, Jaekyoung Lee, Soo Mi Ahn, Jaeho Yeon, ${ }^{\dagger}$ Dae Sung Yoo, ${ }^{\dagger}$ Duck Hee Kim, \\ Han Gon Kim, and Jae Youl Cho ${ }^{\S, *}$ \\ AmorePacific R\&D Center, Yongin, Korea. *E-mail: thiocarbon@freechal.com \\ ${ }^{\dagger}$ AmorePacific R \& D Center, Shanghai, China \\ College of Biomedical Science, Kangwon National University, Korea \\ $\S^{\S}$ Department of Genetic Engineering, Sungkyunkwan University, Korea. ${ }^{*}$ E-mail: jaecho@skku.edu
}

Received August 23, 2010, Accepted December 20, 2010

Key Words : Kojic acid, Polyphenol, Adamantane, Tyrosinase, Nitric oxide

Nitric oxide (NO) is an important inflammatory mediator, synthesized by inducible nitric oxide synthase (iNOS). ${ }^{1}$ Over production of NO can lead to inflammatory diseases. Thus, much effort has been focused on finding potent inhibitors of NO production from natural sources ${ }^{2}$ or developing them by modifying natural products. ${ }^{3}$ Tyrosinase, ${ }^{4}$ a copper-containing enzyme, catalyzes two distinct reactions involved in melanin biosynthesis: hydroxylation of tyrosine to DOPA and oxidation of DOPA to dopaquinone. Three types of tyrosinase (met-, oxy-, and deoxytyrosinase) with different binuclear copper structures at the active site are involved. ${ }^{5}$ Kojic acid (5-hydroxy2-(hydroxymethyl)- $4 H$-pyran-4-one) (1) is one of the metabolites used in fermentation by the species Aspergillus. ${ }^{6}$ Kojic acid inhibits tyrosinase via chelation to copper, which is necessary for tyrosinase inhibitory activity. ${ }^{7}$ Because of these effects, kojic acid has been used as a depigmentation agent in cosmetics 8 and an antibrowning agent in food. ${ }^{9}$ Kojic acid has also been shown to scavenge free radicals and prevent photodamage. ${ }^{10}$ However, there have been few studies on the use of kojic acid and its derivatives as NO inhibitors. ${ }^{11}$ The biological activities of kojic acid are attributed to its $\gamma$-pyranone structure that contains an enolic hydroxyl group. If the enolic hydroxyl group is protected, its activity is completely lost. ${ }^{12}$ Thus, various kojic acid derivatives modified at the 2-position have been developed with enhanced biological activities. ${ }^{13}$ Recently, we reported that the 3,4-methylenedioxycinnamic acid ester of kojic acid (4c) exhibits more potent inhibitory effects on tyrosinase than
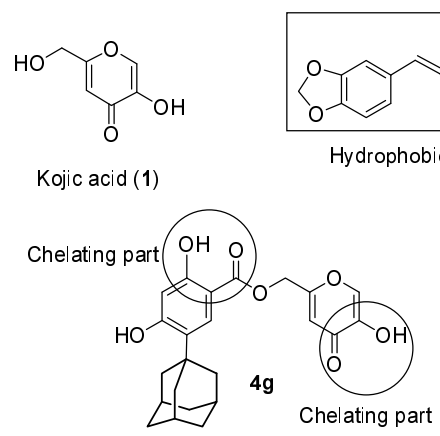

Kojic acid (1)

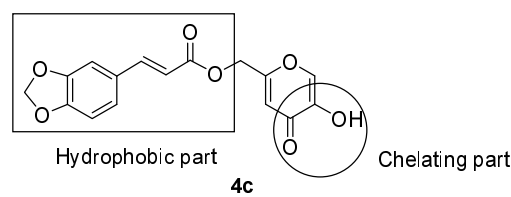

$4 c$

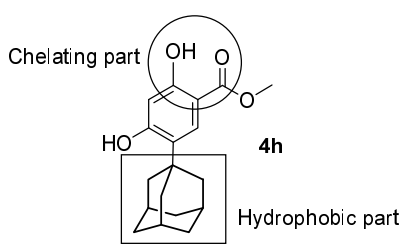

Figure 1. Structures of kojic acid and 2,4-dihydroxy benzoic acid derivatives. kojic acid. ${ }^{14}$ The structure of compound $\mathbf{4 c}$ comprises two main parts: a chelating part of kojic acid and a hydrophobic part of cinnamic acid (Figure 1). In this study on ester derivatives of kojic acid, we synthesized more derivatives (4a-4e). Next, we designed new kojic acid derivatives (4f and $\mathbf{4 g}$ ) containing 2-hyroxy benzoic acid and adamantane moieties. The 2-hydroxy benzoic acid moiety can chelate to copper like the enolic hydroxyl group of kojic acid. Compounds $\mathbf{4 f}$ and $\mathbf{4 g}$ are composed of two copper chelating moieties at different positions. 5-Adamantyl-2,4-dihydroxybenzoic acid derivatives (4h and $\mathbf{4 i}$ ) were also synthesized. Each of them has one copper chelating moiety and an adamantane group. We evaluated the inhibitory activities of the kojic acid derivatives and 2,4-dihydroxy benzoic acid derivatives against tyrosinase and NO production in lipopolysaccharide (LPS) activated macrophages to determine structures important for inhibition.

The synthetic pathways are shown in Schemes 1 and 2. The reaction of kojic acid with thionyl chloride produces compound $\mathbf{2}$, which is conveniently converted to compound $\mathbf{3}$ using dimethylsulfate and potassium carbonate in acetone under reflux conditions. Chlorides $\mathbf{2}$ and $\mathbf{3}$ react with potassium salts of benzoic acids or of cinnamic acids in dimethylformamide at 110 ${ }^{\circ} \mathrm{C}-120^{\circ} \mathrm{C}$ to give the corresponding ester derivatives $4 \mathbf{a}-\mathbf{4 e}$.

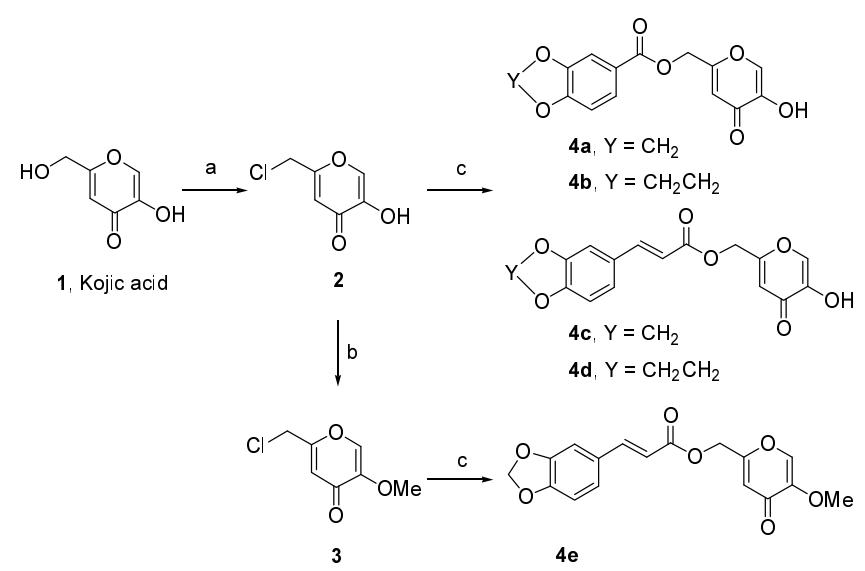

Scheme 1. Reaction conditions: (a) thionyl chloride, DMF, room temperature; (b) dimethylsulfate, potassium carbonate, acetone, reflux; (c) potassium salts of benzoic acids or of cinnamic acids, DMF, 110 ${ }^{\circ} \mathrm{C}-120{ }^{\circ} \mathrm{C}$ 


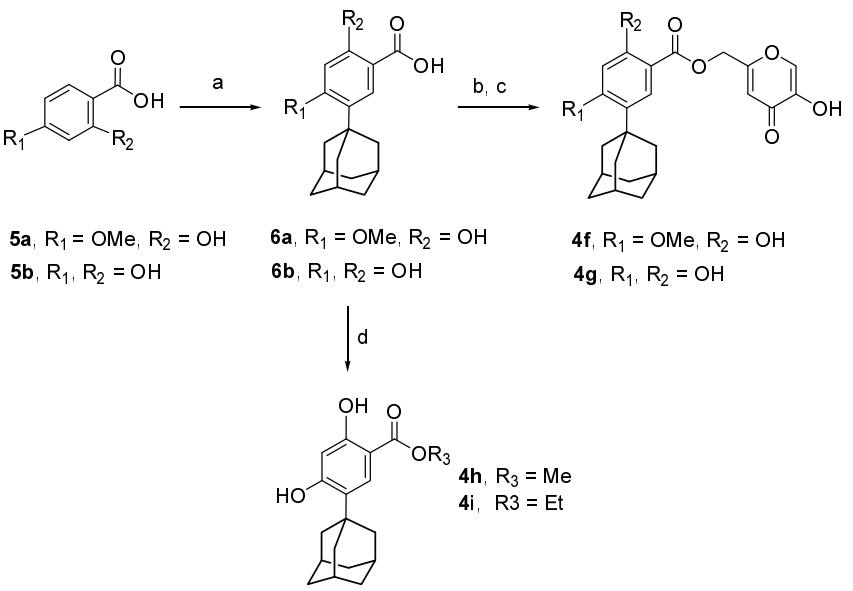

Scheme 2. Reaction conditions: (a) 1-adamantanol, TFA, reflux; (b) potassium hydroxide, methanol, room temperature; (c) kojyl chloride, DMF, $110{ }^{\circ} \mathrm{C}-120{ }^{\circ} \mathrm{C}$; (d) methanol or ethanol, toluene, $\mathrm{H}_{2} \mathrm{SO}_{4}$, reflux

Adamantylbenzoic acids $6 \mathbf{a}$ and $\mathbf{6 b}$ were synthesized by reacting benzoic acids $\mathbf{5} \mathbf{a}$ and $\mathbf{5 b}$ with 1 -adamantanol in trifluoroacetic acid (TFA) under reflux conditions. ${ }^{15}$ Adamantylbenzoic acids $6 \mathbf{a}$ and $\mathbf{6 b}$ react with potassium hydroxide in methanol to afford the potassium salts. After methanol removal, the potassium salts react with kojyl chloride 2 to produce the corresponding ester derivatives $\mathbf{4 f}$ and $\mathbf{4 g}$. Methyl or ethyl esters of 5-adamantly-2,4-dihydroxybenzoic acid (4h and $4 \mathbf{i})$ were synthesized by reacting 5-adamantly-2,4-dihydroxybenzoic acid 6b with methanol or ethanol and toluene in the presence of $\mathrm{H}_{2} \mathrm{SO}_{4}$ under reflux conditions.

First, we evaluated the tyrosinase inhibitory activities ${ }^{16}$ of the kojic acid and 2,4-dihydroxybenzoic acid derivatives. Compounds $\mathbf{4 a - 4 d}$ exhibited potent inhibitory activities against tyrosinase. The hydrophobic benzoate or cinnamate groups increased the inhibitory activity of kojic acid. Compound $\mathbf{4 c}$ showed the most potent inhibitory activity $\left(\mathrm{IC}_{50}=1.52 \mu \mathrm{M}\right)$. Its potency was 14-fold higher than that of kojic acid $\left(\mathrm{IC}_{50}=20.97 \mu \mathrm{M}\right)$. When the enolic hydroxyl group of compound $4 \mathbf{c}$ was protected by a methyl group, the activity was lost completely. These results indicated that the kojic acid moiety may have blocked the copper active site of tyrosinase, and the hydrophobic cinnamate group may have been bound to a secondary binding domain of tyrosinase. However, compounds $\mathbf{4 f}$ and $\mathbf{4 g}$ containing 2-hyroxy benzoic acid and the adamantane and kojic acid moieties showed no tyrosinase inhibitory activity. Although they contained two copper chelating moieties, the bulky adamantane moiety caused steric hindrance and interrupted their binding to the active site of tyrosinase. Interestingly, the methyl ester of 2,4-dihydroxybenzoic acid containing an adamantane moiety (4h) showed mild inhibitory activity $\left(\mathrm{IC}_{50}=33.93 \mu \mathrm{M}\right)$ without the kojic acid moiety, whereas the ethyl ester derivative (4i) showed no activity. In the case of the ester derivatives of 2,4dihydroxy benzoic acids containing an adamantane moiety, the size of the alcohol was a key parameter affecting their activities. From these results, we conclude that 2-hydroxy benzoic acid moiety can be used as an effective functional group for inhibition of tyrosinase.
Table 1. Tyrosinase and NO inhibitory activities of kojic acid derivatives

\begin{tabular}{cccc}
\hline \multirow{2}{*}{ Compounds } & \multicolumn{3}{c}{ Inhibitory activity $\left[\operatorname{IC}_{50}{ }^{a}(\mu \mathrm{M})\right]$} \\
\cline { 2 - 4 } & Tyrosinase & NO & Cytotoxicity \\
\hline $\mathbf{4 a}$ & $3.29 \pm 0.35$ & $>100$ & $>100$ \\
$\mathbf{4 b}$ & $2.77 \pm 0.92$ & $>100$ & $>100$ \\
$\mathbf{4 c}$ & $1.52 \pm 0.58$ & $>100$ & $>100$ \\
$\mathbf{4 d}$ & $1.74 \pm 0.86$ & $>100$ & $>100$ \\
$\mathbf{4 e}$ & - & $>100$ & $>100$ \\
$\mathbf{4 f}$ & - & $54.57 \pm 0.88$ & $28.51^{b}$ \\
$\mathbf{4 g}$ & - & $17.76 \pm 0.49$ & $39.03^{c}$ \\
$\mathbf{4 h}$ & $33.93 \pm 0.45$ & $16.65 \pm 0.48$ & 40.23 \\
$\mathbf{4 i}$ & - & $8.81 \pm 0.18$ & 25.90 \\
Kojic acid & $20.97 \pm 0.74$ & $89.41 \pm 0.24$ & $>100$ \\
\hline
\end{tabular}

${ }^{a}$ Values were determined from logarithmic concentration-inhibition curves and are the means of three experiments. ${ }^{b}$ Cell viability (\% of control) at $100 \mu \mathrm{M}$. ${ }^{c}$ Cell viability (\% of control) at $25 \mu \mathrm{M}$. - : no effect.

After evaluating the tyrosinase inhibitory activity, we investigated the anti-inflammatory activities of kojic acid derivatives and 2,4-dihydroxybenzoic acid derivatives against NO production induced by LPS in macrophages and against cytotoxicity. ${ }^{17}$ The inhibitory activity against NO production differed from that against tyrosinase. Specifically, compounds 4a-4d, which exhibited the highest activity in tyrosinase inhibition, showed no inhibitory activity against NO production. Compounds $\mathbf{4 f}$ and $\mathbf{4 g}$, which exhibited no inhibitory activity against tyrosinase, showed potent inhibitory activities along with cytotoxicity. However, compounds $\mathbf{4 h}$ and $\mathbf{4 i}$ showed potent activities with low cytotoxicity. Taken together, these results suggest that the enolic hydroxyl group of kojic acid and appropriate hydrophobic groups, such as benzoate and cinnamate, are important for the inhibition of tyrosinase. However, the enolic hydroxyl group of kojic acid may not bind to the active site of tyrosinase together with the 2-hydroxy benzoic acid and bulky adamantane moieties. For inhibition of NO production, the 2,4-dihydroxybenzoate moiety can be considered more important pharmacophore than kojic acid.

In conclusion, we synthesized a series of kojic acid derivatives containing ester linkages (4a-4g) and polyphenols containing adamantane moieties ( $2 \mathbf{h}$ and $\mathbf{2 i}$ ) and evaluated their inhibitory activities against tyrosinase and NO production. In the tyrosinase assay, the cinnamate or benzoate ester of kojic acid showed potent inhibitory activity. However, the hydroxybenzoate derivatives of kojic acid containing the adamantane moiety showed no inhibitory activity. The reason for no inhibition may be either steric hindrance of the adamantane moiety or sufficient copper chelating tendency between kojic acid and the 2-hydroxy benzoic acid moiety. A slightly different experimental result was obtained when inhibitory activities against NO production were induced by LPS. Methyl or ethyl esters of 2,4-dihydroxybenzoic acid containing an adamantane moiety showed potent inhibitory activity. Further synthesis of hydroxyl benzoic acid derivatives without the adamantane moiety and studies on their inhibitory activities against tyrosinase and NO production are underway. 


\section{Experimental Section}

(5-Hydroxy-4-oxo-4H-pyran-2-yl)methyl benzo[ $d][1,3]$ dioxole-5-carboxylate (4a). To a stirred solution of kojyl chloride $2(4.8 \mathrm{~g}, 30 \mathrm{mmol})$ in DMF $(100 \mathrm{~mL})$ under $\mathrm{N}_{2}$ was added sodium salt of 3,4-methylenedioxybenzoic acid (7.5 g, $40 \mathrm{mmol})$. The reaction mixture was stirred for $1 \mathrm{~h}$ at $110{ }^{\circ} \mathrm{C}-120{ }^{\circ} \mathrm{C}$, after which DMF was evaporated in vacuo. The residue was extracted with ethyl acetate $(500 \mathrm{~mL})$, washed with water. The organic layer was dried with anhydrous $\mathrm{MgSO}_{4}$ and concentrated to give a crude product. The resultant was purified by crystallization from ethyl acetate-hexane to give $\mathbf{4 a}(6.6 \mathrm{~g})$ in $72 \%$ yields.

${ }^{1} \mathrm{H}$ NMR $\left(300 \mathrm{MHz}, \mathrm{DMSO}-d_{6}\right) \delta 9.23(\mathrm{~s}, 1 \mathrm{H}), 8.11(\mathrm{~s}, 1 \mathrm{H})$, $7.63(\mathrm{~d}, 1 \mathrm{H}, J=8.1 \mathrm{~Hz}), 7.43(\mathrm{~s}, 1 \mathrm{H}), 7.07(\mathrm{~d}, 1 \mathrm{H}, J=8.1 \mathrm{~Hz})$, $6.54(\mathrm{~s}, 1 \mathrm{H}), 6.16(\mathrm{~s}, 2 \mathrm{H}), 5.17(\mathrm{~s}, 2 \mathrm{H}) .{ }^{13} \mathrm{C}-\mathrm{NMR}(125 \mathrm{MHz}$, DMSO- $\left.d_{6}\right) \delta 173.5,164.3,161.5,151.9,147.7,146.0,139.9$, 125.4, 122.4, 112.4, 108.7, 108.3, 102.2, 61.8. FAB MS: (m/e) $291[\mathrm{M}+1]^{+}$

(5-Hydroxy-4-oxo-4H-pyran-2-yl)methyl 2,3-dihydrobenzo [b][1,4]dioxine-6-carboxylate (4b). ${ }^{1} \mathrm{H}$ NMR (300 MHz, DMSO$\left.d_{6}\right) \delta 9.24(\mathrm{~s}, 1 \mathrm{H}), 8.11(\mathrm{~S}, 1 \mathrm{H}), 7.49(\mathrm{~d}, 1 \mathrm{H}, J=8.1 \mathrm{~Hz}), 7.44$ $(\mathrm{s}, 1 \mathrm{H}), 7.01(\mathrm{~d}, 1 \mathrm{H}, J=8.1 \mathrm{~Hz}), 6.52(\mathrm{~s}, 1 \mathrm{H}), 5.17(\mathrm{~s}, 2 \mathrm{H}), 4.32$ $(\mathrm{m}, 4 \mathrm{H}) .{ }^{13} \mathrm{C}-\mathrm{NMR}\left(125 \mathrm{MHz}, \mathrm{DMSO}-d_{6}\right) \delta 173.5,164.3,161.5$, 148.2, 146.0, 143.2, 139.9, 123.1, 121.5, 118.1, 117.3, 112.4, 64.5, 63.8, 61.7. FABMS, $m / e 305[\mathrm{M}+\mathrm{H}]^{+}$

(E)-(5-Hydroxy-4-oxo-4H-pyran-2-yl)methyl 3-(benzo[d] [1,3]dioxol-5-yl)acrylate (4c). ${ }^{1} \mathrm{H}$ NMR (300 MHz, DMSO- $d_{6}$ ) $\delta 9.29(\mathrm{~s}, 1 \mathrm{H}), 8.12(\mathrm{~s}, 1 \mathrm{H}), 7.68(\mathrm{~d}, 1 \mathrm{H}, J=16.2 \mathrm{~Hz}), 7.46$ (s, $1 \mathrm{H}), 7.25(\mathrm{~d}, 1 \mathrm{H}, J=8.1 \mathrm{~Hz}), 6.98(\mathrm{~d}, 1 \mathrm{H}, J=8.1 \mathrm{~Hz}), 6.64(\mathrm{~d}$, $1 \mathrm{H}, J=16.2 \mathrm{~Hz}), 6.52(\mathrm{~s}, 1 \mathrm{H}), 6.08(\mathrm{~s}, 2 \mathrm{H}), 5.07(\mathrm{~s}, 2 \mathrm{H})$. ${ }^{13} \mathrm{C}-\mathrm{NMR}\left(125 \mathrm{MHz}, \mathrm{DMSO}-d_{6}\right) \delta 173.5,165.6,161.6,149.5$, 148.0, 146.0, 145.7, 139.8, 128.2, 125.3, 114.6, 112.5, 108.4, 106.7, 101.6, 61.2. FABMS, $m / e 317[\mathrm{M}+\mathrm{H}]^{+}$.

(E)-(5-Hydroxy-4-oxo-4H-pyran-2-yl)methyl 3-(2,3-dihydrobenzo $[\boldsymbol{b}][1,4]$ dioxin-6-yl)acrylate (4d). ${ }^{1} \mathrm{H}$ NMR (300 MHz, DMSO- $\left.d_{6}\right) \delta 9.24(\mathrm{~s}, 1 \mathrm{H}), 8.11(\mathrm{~s}, 1 \mathrm{H}), 7.65$ (d, $1 \mathrm{H}, J=$ $16.2 \mathrm{~Hz}), 7.32(\mathrm{~s}, 1 \mathrm{H}), 7.26(\mathrm{~d}, 1 \mathrm{H}, J=8.1 \mathrm{~Hz}), 6.90(\mathrm{~d}, 1 \mathrm{H}, J=$ $8.1 \mathrm{~Hz}), 6.60(\mathrm{~d}, 1 \mathrm{H}, J=16.2 \mathrm{~Hz}), 6.51(\mathrm{~s}, 1 \mathrm{H}), 5.06(\mathrm{~s}, 2 \mathrm{H})$, $4.27(\mathrm{~m}, 4 \mathrm{H}) .{ }^{13} \mathrm{C}-\mathrm{NMR}\left(125 \mathrm{MHz}, \mathrm{DMSO}-d_{6}\right) \delta 173.5,165.6$, 161.6, 146.00, 145.84, 145.56, 143.5, 139.8, 127.2, 122.4, 117.4, 117.0, 114.7, 112.5, 64.3, 63.8, 61.2. FABMS, $m / e 331[\mathrm{M}+\mathrm{H}]^{+}$.

(E)-(5-Methoxy-4-oxo-4H-pyran-2-yl)methyl 3-(benzo[d] [1,3]dioxol-5-yl)acrylate (4e). ${ }^{1} \mathrm{H}$ NMR $\left(300 \mathrm{MHz}, \mathrm{DMSO}-d_{6}\right.$ ) $\delta 8.13(\mathrm{~s}, 1 \mathrm{H}), 7.68(\mathrm{~d}, 1 \mathrm{H}, J=16.2 \mathrm{~Hz}), 7.45(\mathrm{~s}, 1 \mathrm{H}), 7.24(\mathrm{~d}$, $1 \mathrm{H}, J=8.1 \mathrm{~Hz}), 6.97(\mathrm{~d}, 1 \mathrm{H}, J=8.1 \mathrm{~Hz}), 6.63(\mathrm{~d}, 1 \mathrm{H}, J=16.2$ $\mathrm{Hz}), 6.48$ (s, 1H), 6.08 (s, 2H), 5.07 (s, 2H), 3.65 (s, 3H). ${ }^{13} \mathrm{C}-\mathrm{NMR}\left(125 \mathrm{MHz}, \mathrm{DMSO}-d_{6}\right) \delta 172.4,165.6,161.6,149.5$, 148.14, 148.04, 145.7, 139.5, 128.2, 125.3, 114.6, 113.4, 108.4, 106.7, 101.6, 61.1, 56.2. FABMS, $m / e 331[\mathrm{M}+\mathrm{H}]^{+}$.

(5-Hydroxy-4-oxo-4H-pyran-2-yl)methyl 2-hydroxy-4-methoxy-5-adamanylbenzoate (4f). ${ }^{1} \mathrm{H}$ NMR (300 MHz, DMSO$\left.d_{6}\right) \delta 10.36(\mathrm{~s}, 1 \mathrm{H}), 9.29(\mathrm{~s}, 1 \mathrm{H}), 8.12(\mathrm{~s}, 1 \mathrm{H}), 7.55(\mathrm{~s}, 1 \mathrm{H}), 6.55$ $(\mathrm{s}, 1 \mathrm{H}), 6.53(\mathrm{~s}, 1 \mathrm{H}), 5.22(\mathrm{~s}, 2 \mathrm{H}), 3.84(\mathrm{~s}, 3 \mathrm{H}), 1.97(\mathrm{~s}, 9 \mathrm{H})$, $1.70(\mathrm{~s}, 6 \mathrm{H}) .{ }^{13} \mathrm{C}-\mathrm{NMR}\left(125 \mathrm{MHz}, \mathrm{DMSO}-d_{6}\right) \delta 173.5,167.8$, 164.8, 161.44, 161.03, 146.0, 139.9, 129.9, 127.2, 112.5, 103.6, $100.4,61.6,55.5,36.3,35.8,28.2$. FABMS, $m / e 427[\mathrm{M}+\mathrm{H}]^{+}$.

(5-Hydroxy-4-oxo-4H-pyran-2-yl)methyl 2,4-dihydroxy- 5-adamantylbenzoate (4g). ${ }^{1} \mathrm{H}$ NMR $\left(300 \mathrm{MHz}, \mathrm{DMSO}-d_{6}\right) \delta$ $10.59(\mathrm{~s}, 1 \mathrm{H}), 10.22(\mathrm{~s}, 1 \mathrm{H}), 9.26(\mathrm{~s}, 1 \mathrm{H}), 8.12(\mathrm{~s}, 1 \mathrm{H}), 7.51(\mathrm{~s}$, $1 \mathrm{H}), 6.51(\mathrm{~s}, 1 \mathrm{H}), 6.36(\mathrm{~s}, 1 \mathrm{H}), 5.19(\mathrm{~s}, 2 \mathrm{H}), 2.00(\mathrm{~s}, 9 \mathrm{H}), 1.70(\mathrm{~s}$, 6H). ${ }^{13} \mathrm{C}-\mathrm{NMR}\left(125 \mathrm{MHz}\right.$, DMSO- $\left.d_{6}\right) \delta 173.5,167.8,163.5$, 161.5, 146.0, 139.9, 128.8, 127.9, 117.3, 112.4, 103.6, 101.2, 61.4, 36.4, 35.6, 28.2. FABMS, $m / e 413[\mathrm{M}+\mathrm{H}]^{+}$.

Methyl 2,4-Dihydroxy-5-adamantylbenzoate (4h). ${ }^{1} \mathrm{H}$ NMR $\left(300 \mathrm{MHz}, \mathrm{DMSO}-d_{6}\right) \delta 10.4$ (bs, 2H), $7.50(\mathrm{~s}, 1 \mathrm{H}), 6.34(\mathrm{~s}, 1 \mathrm{H})$, $3.83(\mathrm{~s}, 3 \mathrm{H}), 2.00(\mathrm{~s}, 9 \mathrm{H}), 1.70(\mathrm{~s}, 6 \mathrm{H}) .{ }^{13} \mathrm{C}-\mathrm{NMR}(125 \mathrm{MHz}$, DMSO- $\left.d_{6}\right) \delta 169.6,163.2,160.3,128.6,127.5,103.44,103.07$, $51.8,36.5,35.5,28.2$. FABMS, $m / e 303[\mathrm{M}+\mathrm{H}]^{+}$.

Ethyl 2,4-Dihydroxy-5-adamantylbenzoate (4i). ${ }^{1} \mathrm{H}$ NMR (300 MHz, DMSO- $\left.d_{6}\right) \delta 10.5(\mathrm{~s}, 1 \mathrm{H}), 10.40(\mathrm{~s}, 1 \mathrm{H}), 7.50$ (s, $1 \mathrm{H}), 6.33(\mathrm{~s}, 1 \mathrm{H}), 4.32(\mathrm{~m}, 2 \mathrm{H}), 2.00(\mathrm{~s}, 9 \mathrm{H}), 1.70(\mathrm{~s}, 6 \mathrm{H}), 1.33$ $(\mathrm{t}, 3 \mathrm{H}, J=6.9 \mathrm{~Hz}) .{ }^{13} \mathrm{C}-\mathrm{NMR}\left(125 \mathrm{MHz}\right.$, DMSO- $\left.d_{6}\right) \delta 169.4$, 163.2, 160.5, 128.6, 127.4, 103.40, 103.16, 60.5, 36.4, 35.6, 28.2, 14.11. FABMS, $m / e 317[\mathrm{M}+\mathrm{H}]^{+}$.

Mushroom Tyrosinase Assay. Mushroom tyrosinase, L-tyrosine were purchased from Sigma Chemical. The reaction mixture for mushroom tyrosinase activity consisted of $150 \mu \mathrm{L}$ of $0.1 \mathrm{M}$ phosphate buffer ( $\mathrm{pH} 6.5$ ), $3 \mu \mathrm{L}$ of sample solution, $8 \mu \mathrm{L}$ of mushroom tyrosinase $(2,100 \mathrm{unit} / \mathrm{mL}, 0.05 \mathrm{M}$ phosphate buffer at $\mathrm{pH} 6.5$ ), and $36 \mu \mathrm{L}$ of $1.5 \mathrm{mM}$ L-tyrosine. Tyrosinase activity was determined by reading the optical density at $490 \mathrm{~nm}$ on a microplate reader (Bio-Rad 3550, Richnmond, CA, U.S.A.) after incubation for $20 \mathrm{~min}$ at $37^{\circ} \mathrm{C}$. The inhibitory activity of the sample was expressed as the concentration that inhibits $50 \%$ of the enzyme activity $\left(\mathrm{IC}_{50}\right)$.

Measurements of NO Production. RAW264.7 cells $\left(1 \times 10^{6}\right.$ cells $/ \mathrm{mL}$ ) were preincubated with kojic acid derivatives for 30 min and continuously activated with LPS $(1 \mu \mathrm{g} / \mathrm{mL})$ for $24 \mathrm{~h}$. Nitrite in culture supernatants was measured by adding $100 \mu \mathrm{L}$ of Griess reagent (1\% sulfanilamide and $0.1 \% N$-[1-naphthyl]ethylenediamine dihydrochloride in $5 \%$ phosphoric acid) to $100 \mu \mathrm{L}$ samples of the medium for $10 \mathrm{~min}$ at room temperature. $\mathrm{OD}$ at $570 \mathrm{~nm}\left(\mathrm{OD}_{570}\right)$ was measured using a Spectramax 250 microplate reader (Molecular Devices, Sunnyvale, CA, U.S.A.). A standard curve of $\mathrm{NO}$ was made with sodium nitrite.

Measurements of Cytotoxicity. After the preincubation of RAW264.7 cells $(1 \times 106$ cells $/ \mathrm{mL})$ for $18 \mathrm{~h}$, kojic acid derivatives $(0-100 \mu \mathrm{M})$ were added to the cells and incubated for $24 \mathrm{~h}$. The cytotoxic effect of kojic acid derivatives was then evaluated by a conventional MTT assay. At $3 \mathrm{~h}$ prior to culture termination, $10 \mu \mathrm{L}$ of the MTT solution $(5 \mathrm{mg} / \mathrm{mL}$ in a phosphate buffered-saline, $\mathrm{pH}$ 7.4) were added and the cells were continuously cultured until termination. The incubation was halted by the addition of $15 \%$ sodium dodecyl sulfate into each well, solubilizing formazan. The absorbance at $570 \mathrm{~nm}$ (OD ${ }_{570-630)}$ was measured by a Spectramax 250 microplate reader.

\section{References and Notes}

1. (a) Kanwar, J. R.; Kanwar, R. K.; Burrow, H.; Baratchi, S. Cur. Med. Chem. 2009, 16, 2373. (b) Hesslinger, C.; Strub, A.; Boer, R.; Ulrich, W. R.; Lehner, M. D.; Braun, C. Biochem. Soc. Trans. 2009, 37, 886 .

2. (a) Yu, T.; Lee, J.; Lee, Y. G.; Byeon, S. E.; Kim, M. H.; Sohn, E.-H.; Lee, Y. J.; Lee, S. G.; Cho, J. Y. Journal of Ethnopharmacology 
2010, 128, 139. (b) Chin, Y.-W.; Chae, H.-S.; Lee, J.; Bach, T. T.; Ahn, K.-S.; Lee, H.-K.; Joung, H.; Oh, S.-R. Bull. Korean Chem. Soc. 2010, 31, 2665.

3. Hwang, J. W.; Choi, D. H.; Joen, J.-H.; Kim, J.-K.; Jun, J.-G. Bull. Korean Chem. Soc. 2010, 31, 965.

4. Marmol, V. D.; Beermann, F. FEBS Lett. 1996, 381, 165.

5. Fenoll, L. G.; Rodriguez-Lopez, J. N.; Garcia-Sevilla, F.; GarciaRuiz, P. A.; Varon, R.; Garcia-Canovas, F.; Tudela, J. Biochim. Biophys. Acta 2001, 1548, 1.

6. Manabe, M.; Goto, T.; Tanaka, K.; Matsuura, S. Rep. Natl. Food Res. Inst. 1981, 38, 115.

7. (a) Yuen, V. G.; Caravan, P.; Gelmini, L.; Glover, N.; Mcneill, J. H.; Setyawati, I. A.; Zhou, Y.; Orvig, C. J. Inorg. Biochem. 1997, 73, 109. (b) Saruno, R.; Kato, F.; Ikeno, T. Agric. Biol. Chem. 1979, 43, 1337.

8. Ohyama, Y.; Mishima, Y. Fragrance J. 1990, 6, 53.

9. Chen, J. S.; Wei, C. I.; Rolle, R. S.; Otwell, W. S.; Balaban, M. D.; Marshall, M. R. J. Agric. Food. Chem. 1991, 39, 1396.

10. (a) Mitani, H.; Koshiishi, I.; Sumita, T.; Imanari, T. Eur. J. Pharmacol. 2001, 411, 169. (b) Kim, M. S.; Lee, S.; Rho, H. S.; Kim, D. H.; Chang, I. S.; Chung, J. H. Clinica. Chimica. Acta 2005, 362, 161.

11. (a) Yoo, D. S.; Lee, J.; Choi, S. S.; Rho, H. S.; Cho, D. H.; Shin,
W. C.; Cho, J. Y. Pharmazie 2010, 65, 261. (b) Rho, H. S.; Ahn, S. M.; Yoo, D. S.; Kim, M. K.; Cho, D. H.; Cho, J. Y. Bioorg. Med. Chem. Lett. 2010, 20, 6569.

12. Rho, H. S.; Baek, H. S.; Ahn, S. M.; Kim, M. K.; Ghimeray, A. K.; Cho, D. H.; Hwang, J. S. Bull. Korean Chem. Soc. 2010, $31,2375$.

13. (a) Kobayashi, Y.; Kayahara, H.; Tadasa, K.; Tanaka, H. Bioorg. Med. Chem. Lett. 1996, 6, 1303. (b) Rho, H. S.; Baek, H. S.; Ahn, S. M.; Kim, D. H.; Chang, I. S. Bull. Korean Chem. Soc. 2008, 29, 1569. (c) Lee, Y. S.; Park, J. H.; Kim, M. H.; Seo, S. H.; Kim, H. J. Arch. Pharm. Chem. Life. Sci. 2006, 339, 111. (d) Yoon, I. H.; Lee, Y. H.; Park, C. W.; Ji, H.; Lee, Y. S. Bull. Korean Chem. Soc. 2010, 31, 2036.

14. Rho, H. S.; Baek, H. S.; You, J. W.; Kim, S.; Lee, J. Y.; Kim, D. H.; Chang, I. S. Bull. Korean Chem. Soc. 2007, 28, 471.

15. (a) Shokova, E.; Tafeenko, V.; Kovalev, V. Tetrahedron Lett. 2002, 43, 5153. (b) Rho, H. S.; Baek, H. S.; Ahn, S. M.; Yoo, J. W.; Kim, D. H.; Kim, H. G. Bioorg. Med. Chem. Lett. 2009, 19, 1532.

16. Rho, H. S.; Baek, H. S.; Ahn, S. M.; Yoo, J. W.; Kim, D. H.; Kim, H. G. Bull. Korean Chem. Soc. 2009, 30, 475.

17. Byeon, S. E.; Lee, Y. G.; Kim, B. H.; Shen, T.; Lee, S. Y.; Park, H. J.; Park, S. C.; Rhee, M. H.; Cho, J. Y. Microbiol. Biotechnol. 2008, $18,1984$. 